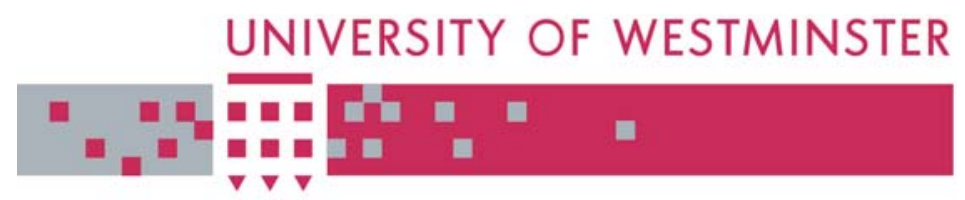

\title{
WestminsterResearch
}

http://www.wmin.ac.uk/westminsterresearch

\section{CAD for broadband wireless access design.}

\section{Djuradj Budimir ${ }^{1}$ \\ B.N. Shelkovnikov ${ }^{2}$}

${ }^{1}$ School of Informatics, University of Westminster

2 Telecommunication Tools Department. NTUU "KPI". 01056, Kiev, Pobedy ave. 37, Ukraine

Copyright (C) [2001] IEEE. Reprinted from the proceedings of 5th International Conference on Telecommunications in Modern Satellite, Cable and Broadcasting Service, 2001 (TELSIKS 2001), pp. 525-528.

This material is posted here with permission of the IEEE. Such permission of the IEEE does not in any way imply IEEE endorsement of any of the University of Westminster's products or services. Internal or personal use of this material is permitted. However, permission to reprint/republish this material for advertising or promotional purposes or for creating new collective works for resale or redistribution must be obtained from the IEEE by writing to pubs-permissions@ieee.org. By choosing to view this document, you agree to all provisions of the copyright laws protecting it.

The WestminsterResearch online digital archive at the University of Westminster aims to make the research output of the University available to a wider audience. Copyright and Moral Rights remain with the authors and/or copyright owners.

Users are permitted to download and/or print one copy for non-commercial private study or research. Further distribution and any use of material from within this archive for profit-making enterprises or for commercial gain is strictly forbidden.

Whilst further distribution of specific materials from within this archive is forbidden, you may freely distribute the URL of the University of Westminster Eprints (http://www.wmin.ac.uk/westminsterresearch).

In case of abuse or copyright appearing without permission e-mail wattsn@wmin.ac.uk. 


\title{
CAD for Broadband Wireless Access Design
}

\author{
Djuradj Budimir ${ }^{1}$, B.N.Shelkovnikov ${ }^{2}$
}

\begin{abstract}
The paper observes CAD of leading companies in the field of wireless broadband systems development. The basic characteristics of appropriate software and design systems are shown including the most typical examples for their implementation.
\end{abstract}

Keywords - CAD, wireless broadband access, design.

\section{INTRODUCTION}

The leading companies, that provide marketing research, predict [1] the huge growth in application of wireless broadband access equipment. This shows the need in CAD for Wireless Broadband System Design, that provides an effective way to design wireless systems, such as LMDS, MMDS, BWS, WLL, and others, which allow to significantly solve the "last mile" problem, allowing for the communication infrastructure to grow.

That is why broadband wireless access (BWA) equipment design is performed on the base of multi-functional and specialized CAD systems and lead to the software and CAD system development.

\section{CAD FOR WIRELESS BROADBAND ACCESS DESIGN}

The tasks for broadband wireless access are solved by sets of different systems, such as "point-to-point" (PtP) or simple radio-relay line, "point-to-multipoint" (PMP), MMDS, LMDS, MVDS and other. Usually, such systems work on microwaves in the frequency range of 2 to $40 \mathrm{GHz}$ and use different, access methods - FDMA, TDMA, CDMA, orthogonal frequency division multiplexing (OFDM) - code (COFDM) and vector (VOFDM). The information flow rate and bandwidth depend on the modulation method used QPSK (4 QAM), ... 64 QAM, 256 QAM and other.

The usual broadband wireless access system is shown on the Fig.1 [2].

It includes the Network Management System or a network node, Base Station, user equipment (CPE Transceiver and other). The network node devices provide multiplexing, channel division, routing, modulation and demodulation,

\footnotetext{
'Djuradj Budimir is with the Wireless Communication Research Groups, Department of Electronic Systems, University of Westminster, London WIW 6UW, UK, E-mail: budimid@wnin.ac.uk

${ }^{2}$ B.N.Shelkovnikov is with the Telecommunication Tools Department, NTUU "KPI", 01056, Kiev, Pobedy ave. 37, Ukraine, E-mail: shelk@ukr.net
}

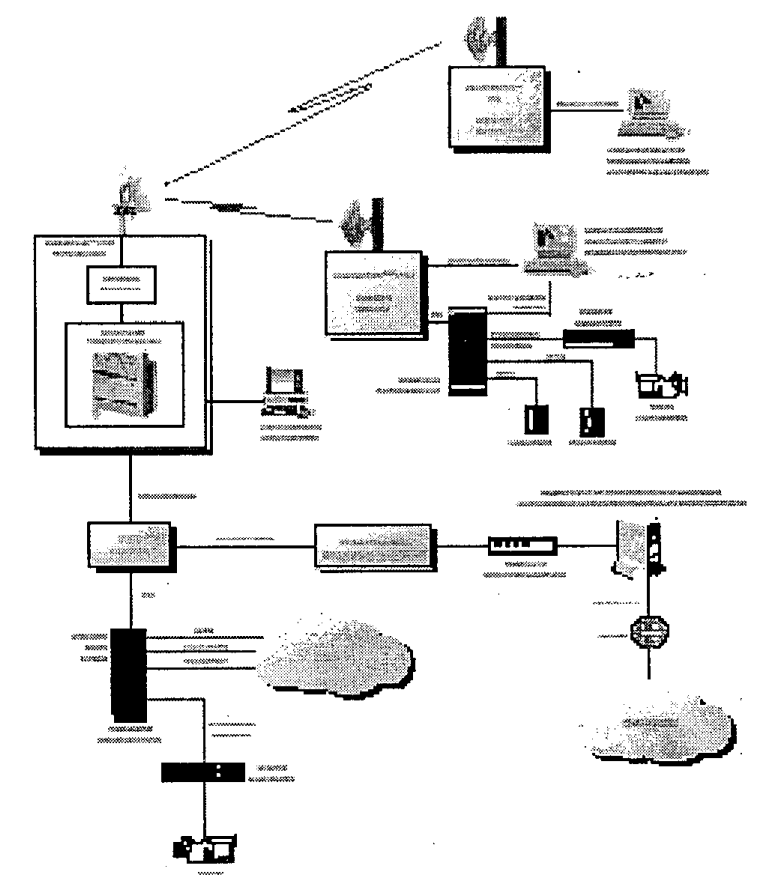

Fig.1 Multi-Service Broadband Wireless Access solution

different protocols support (including ATM). Base Station is a transceiver and an antenna system. . The user equipment, except for the transmitter, can contain network interface modules, which provide the necessary: service to the user data, video and television signal (analog and digital). Internet.

From the large amount of software packages the modern requirements of the WBA design are fulfilled by the software of Ansoft Corparation [3], ICUCOM Corparation [4], Xpedian Design Systems [5], Elanix Inc. [6], Agilent Technologies [7], EDX Division, Comarco Wireless Technologies, Inc. [8].

Ansoft's Serenade Design Environment offers a powerful range of circuit, system, and electromagnetic simulation tools for the modern RF and microwave designer. Windows interface provides an intuitive, easy-to-use environment that allows the user to maximize productivity and facilitates data transfer between the simulator and other tools, such as word processors and presentation software.

Symphony adds efficient analog, digital, and mixed-mode (analog and digital) system analysis capabilities to the Environment. Users can quickly construct a system by including blocks from libraries of built-in analog RF and digital signal processing (DSP) components. Blocks such as channel coders, analog and DSP filters, amplifiers, oscillators, and fading channel models enable engineers to quickly put 
together complete wired and wireless systems. Since these are behavioral models, users need only enter a few key features for each block to begin simulating. Complete linear and nonlinear system analysis is done providing outputs such as gain, noise figure, and bit error rate (BER) both in the time and frequency domains. Trade-offs between different design approaches can be rapidly investigated at an early stage to reduce design cycle time and avoid costly redesigns due to RF and DSP system interactions.

Once a system topology is chosen, a top-down design flow can be achieved. Whether individual blocks are designed at the component level using the Serenade Design Environment, or purchased as ready-made components, measured or simulated data can be brought directly into Symphony (Fig.2 and 3).

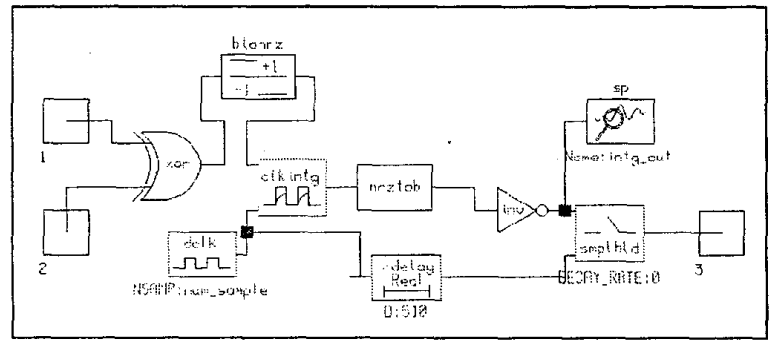

Fig.2. A direct sequence spread spectrum(DSSS) wireless communications transceiver CDMA

The schematic of the demodulation circuit.

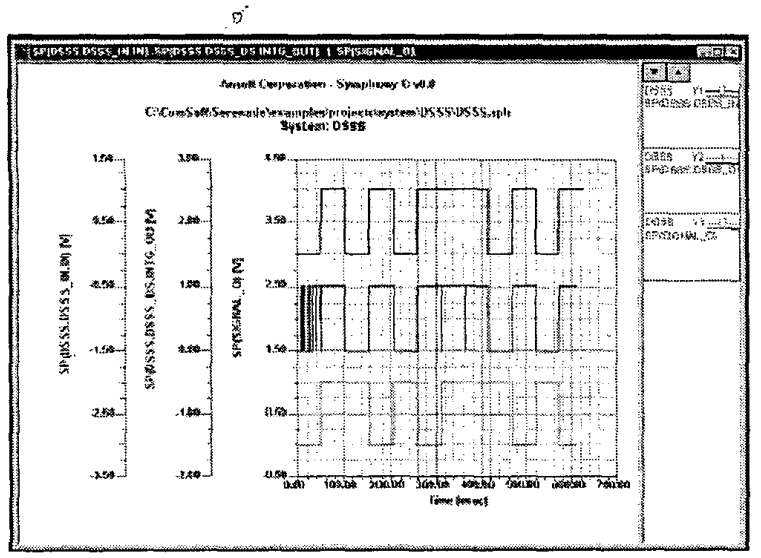

Fig.3. Original input signal, demodulated signal, and delayed demodulated signal.

ACOLADE is a comprehensive system design and simulation tool that allows to design and experiment with complicated communication and DSP systems. ACOLADE provides a flexible environment for communication system study and development that requires no programming. It achieves its flexibility through a modular modeling philosophy, in which the simulation is based on the concept of Models; each Model corresponds to a typical component in a communication system (e.g., antenna, transmitter, receiver, etc.). Models can be interconnected in accordance with any conceivable system architecture. Each one of the ACOLADE
Models contains a set of parameters that governs the operation of that model. These parameters can be accessed and changed to test the effects of these changes on the overall performance of the system. ACOLADE contains an up-to-date model library that includes a wide variety of generic system components along with very specialized and state-of-the-art components, forming the largest collection of models for DSP and communication simulation on the market. This model library is continuously updated to reflect new discoveries and achievements in the industry.

ICUCOM also has several optional libraries available for use with ACOLADE. These include the CDMA, Finite Precision, RF, GSM, GPS and Advanced Analysis libraries.

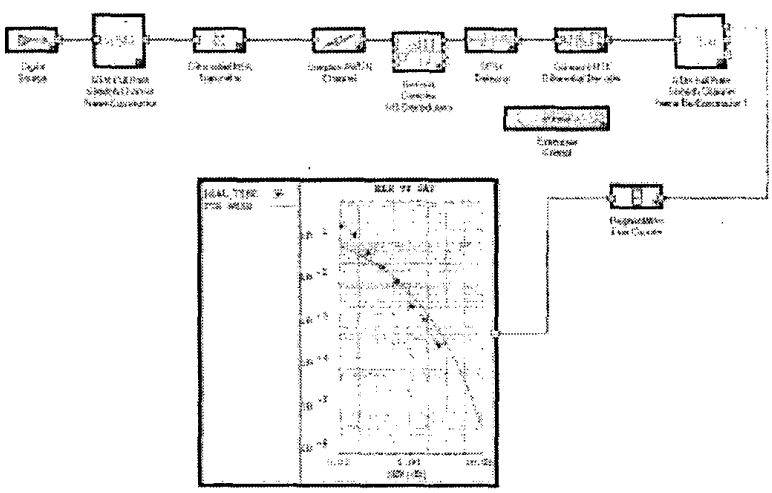

Fig.4. Orthogonal Coherent detection for GMSK

Xpedion GoldenGate RF/Microwave Design Tools offer the functionality that designers need to simulate modern wireless designs, and are integrated into commonly used EDA environments. Xpedion GoldenGate includes advanced RF and microwave simulators- RF linear, RF non-linear harmonic balance with Krylov sub-space and envelope transient with non-linear phase noise. Seamlessly integrated into widely used Affirma Analog Artist EDA environment, GoldenGate is complementary to Spectre RF and significantly reduces design-cycles and design-time for wireless integrated circuits and products.

The GoldenGate/Neural Net Model Compiler (NN-MC), based on patent-pending technology, is an innovative behavior modeling tool to accurately model complex RF and microwave linear and non-linear circuits. Using NN-MC models for the first time, designers can accurately analyze complete transmitter and receiver circuitry with realistic input digital signal stream, without sacrificing circuit-level simulation accuracy. NN-MC generated RF models enable product designers to perform accurate system-partitioning with significant reduction in time-to-market for wireless (Fig.5).

SystemView is a comprehensive dynamic systems analysis environment for the design and simulation of engineering or scientific systems. From analog or digital signal processing, filter design, control systems, and products, communication systems to general mathematical systems modelling. 


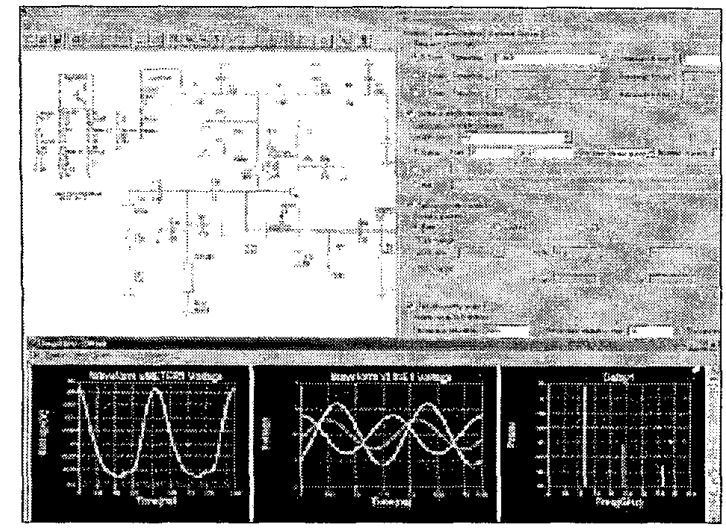

Fig.5. Windows-based Xpedion GoldenGate, integrated with OrCAD schematic capture, is a powerful simulator and display tool

SystemView provides a sophisticated analysis engine. In concert with Microsoft's Windows family of operating systems, SystemView helps to concentrate on what's really important...designing, simulating and documenting you systems rapidly and efficiently. And taking the next step to hardware implementation is a mouse click away with DSP Direct options such as the Xilinx FPGA option.

SystemView consists of two primary windows: the System window and the Analysis window. Design your system in the System window by selecting tokens from various libraries including Sources, Sinks, Operators and Functions. Parameters for each functional block are specified by doubleclicking on the block, then entering values in user-friendly dialog boxes.

Large systems can be easily simplified in SystemView by defining groups of tokens as MetaSystems. A MetaSystem allows a single token to represent a complete system or subsystem. MetaSystem connections are handled in the same manner as any other SystemView token. A simple mouse click opens a window showing the complete subsystem contained in the MetaSystem (Fig.6-7).

SystemView contains all of the tools necessary to develop modern wired and wireless communications systems. Typical applications include PCS/cellular radios (GSM, CDMA, TDMA, pi/4 DQPSK); cordless phones, pagers, and modems (wired and wireless); satellite systems (GPS, DBS, LEOS); and spread-spectrum systems. All of the elements required to perform complete end-to-end system simulations are available. This library includes error-correcting codes, base band pulse shaping (i.e. raised cosine), Gray encoding, modulation and demodulation, channel models, and data recovery. Complex models such as the Reed Solomon (RS) error-correction encoder/decoder, the Costas Loop, and the PLL demodulator are provided as individual functional blocks.

HP Advanced Design System(ADS) was developed to improve communication design process. This system is the culmination of HP EEsof's years of expertise in developing $\mathrm{RF}$, microwave, and system design automation solutions.

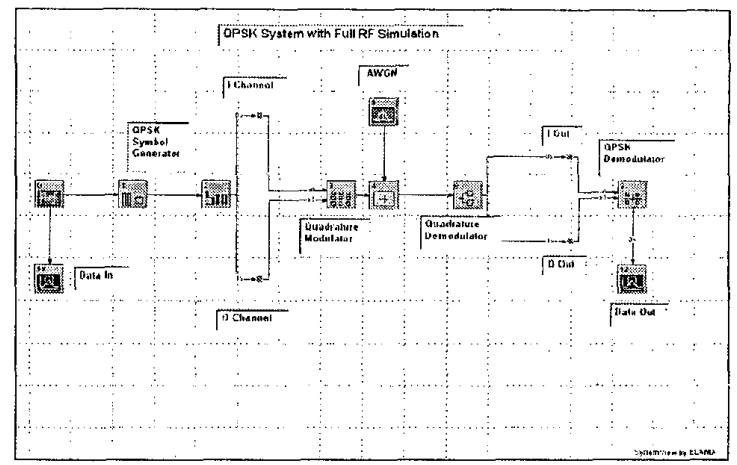

Fig.6. QPSK Simulation with RF Frequency Included

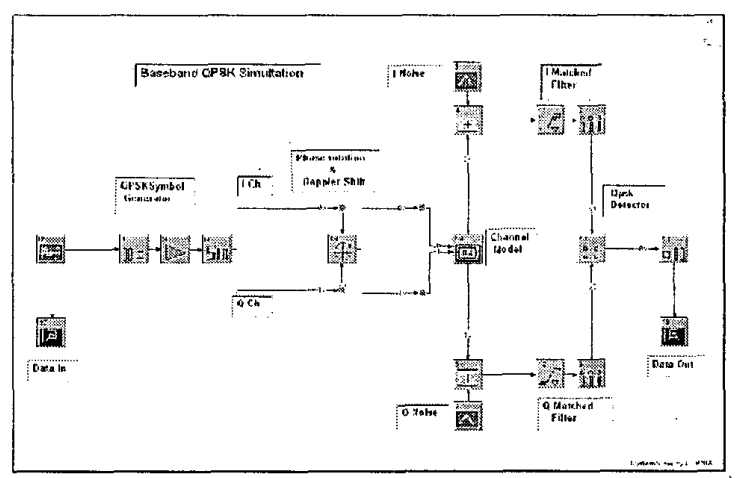

Fig.7. Baseband QPSK Simulation

Complete, Integrated Solutions for End-to-End Communication Signal Path Design HP Advanced Design System is a family of EDA modules that offers designers of communications products a complete signal path solution. The family is composed of EDA modules including circuit and system simulators, translators, layout solutions, models; libraries, etc. The modules have been selectively bundled into design suites that provide solutions to specific problems.

HP offers a variety of suites, bundled to provide solutions to communications system, DSP, RFIC, microwave, RF board, layout, and related design problems.

The following suites are explained in HPADS: HP Communication Systems Designer, HP DSP Designer, HP DSP Synthesis, HP RFIC Designer, HP Microwave Circuit Designer, HP RF Board Designer. HP ADS also offers specialized solutions for high-frequency structure simulation and statistical modeling of ICs and nonlinear devices.

Developed for communication system architects and subsystem designers, this suite lets you model, compare, and trade off architectures, standards, system partitions, specifications, and other factors that affect a system's performance. The suite simplifies the generation of systemlevel and subsystem-level specifications that are not over- or under-specified. This can translate into significant savings of component development time. A typical radio link, for example, has at least an encoder, decoder, modulator, demodulator, up-converter, down-converter, power amplifier, 
low-noise amplifier, and IF section. The minimal benefit from not over-specifying these components could exceed one engineering month. Further, the statistical package (included in Premier) offers guidance on how to maximize manufacturing yield and minimize cost. Finally, the ability to download or upload analog and digital signals to instrumentation leads to better analysis and under-standing of the hardware during the critical integration phase (Fig.8).

There are three versions of this suite:

- HP Communication Systems Designer is tailored for RF system engineers. It contains an RF simulator that accurately predicts performance of complete RF systems. It also includes a set of block-level RF models for linear and non-linear parts. Some functionality is restricted with this version of the suite.

- HP Communication Systems Designer Pro offers RF system modeling and general signal processing, including DSP. In addition, it allows co-simulation between RF system models and DSP simulations. For example, this package allows to implement floating-point DSP algorithms and, in the same simulation, predict system performance measures like BER.

- HP Communication Systems Designer Premier extends the suite's simulation capability to include radio propagation models for GSM, IS-95 CDMA, and IS-54 TDMA. And, with the addition of the Matrix model set, you can create block processing models for data compression methods such as JPEG and MPEG. The matrix models also allow to do bidirectional co-simulation with MATLAB.

HP DSP Designer, HP DSP Synthesis, HP RFIC Designer, HP Microwave Circuit Designer, HP RF Board Designer fulfil the tasks of elements design and the design of layout Communication Systems.

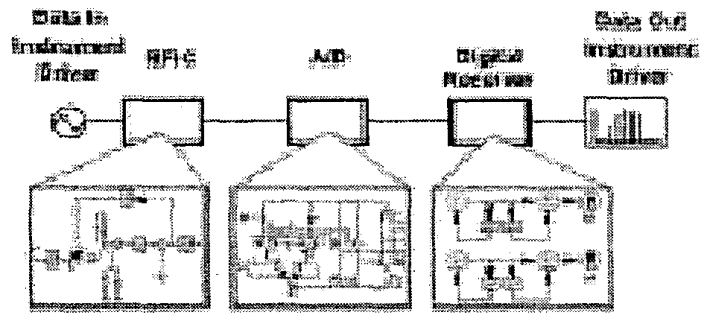

Fig.8. DSP Designer lets you integrate the RF, A-D and DSP components of a system into a unified design flow

EDX Division of Comarco Wireless Technologies, Inc. provides the set of tools for wireless design: EDX SignalPro with the Network Design Module and the Microcell/Indoor Module.

EDX SignalPro is a comprehensive planning tool for LMDS, MMDS, WLL, PCS, cellular, and other wireless communication systems. It uses propagation models to perform coverage, interference and detailed link analysis studies for mobile and fixed wireless networks.

The Network Design Module includes capabilities for automatic system layout and frequency planning with the support of different system types like AMPS, TDMA, GSM and CDMA. For fixed point-to-multipoint networks, the module can develop system layouts with multi-sector hubs and an unlimited number of customer terminals (CTs), each of which can be specified with different directional antennas, power levels, frequencies, modulation schemes, and other. It provides complete two-way analysis, including signal levels, fade margin, and rain outage (Fig.9).

The Microcell/Indoor Module includes ray-tracing propagation modules that consider the location of building walls and corners. This module features outdoor and indoor microcell models for signal level analysis. It also gives a possibility to calculate bit error rates for multiple digital modulation types.

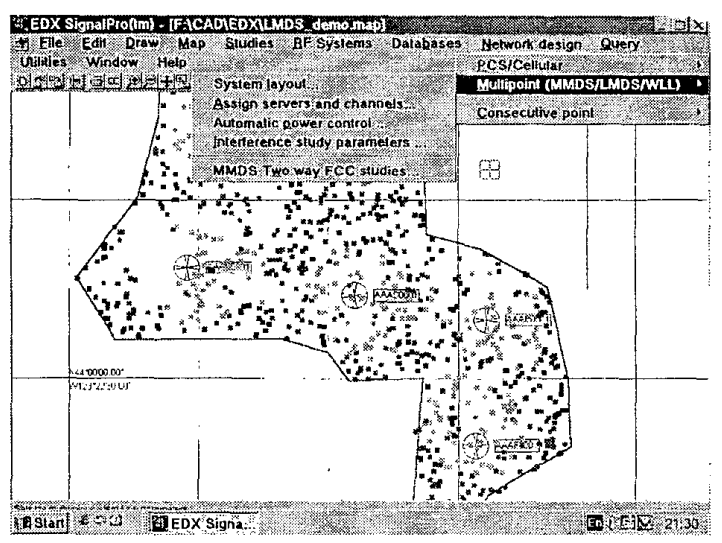

Fig.9. Examles Map LMDS

\section{CONCLUSION}

The common part for the whole CAD BWA is the existence of both text and graphic interfaces, element and graphics editors, database and libraries, information output service. All CAD for BWA not only compete, but add each other, which increases their application domains.

The results of this work can help designers of equipment and wireless broadband systems to choose an effective tool for simulation and design.

\section{REFERENCES}

[1] www. wirelessweek.com

[2] www harris.com

[3] www.ansoft.com

[4] www.icucom.com

[5] www.xpedion.com

[6] www.elanix.com

[7] www.agilent.com

[8] www.edx.com 